\title{
Three-year efficacy, safety, and survival findings from COMFORT-II, a phase 3 study comparing ruxolitinib with best available therapy for myelofibrosis
}

\author{
Francisco Cervantes, ${ }^{1}$ Alessandro M. Vannucchi, ${ }^{2}$ Jean-Jacques Kiladjian, ${ }^{3}$ Haifa Kathrin Al-Ali, ${ }^{4}$ Andres Sirulnik, ${ }^{5}$ \\ Viktoriya Stalbovskaya, ${ }^{6}$ Mari McQuitty, ${ }^{6}$ Deborah S. Hunter, ${ }^{7}$ Richard S. Levy, ${ }^{7}$ Francesco Passamonti, ${ }^{8}$ Tiziano Barbui, ${ }^{9}$ \\ Giovanni Barosi, ${ }^{10}$ Claire N. Harrison, ${ }^{11}$ Laurent Knoops, ${ }^{12}$ and Heinz Gisslinger, ${ }^{13}$ on behalf of the COMFORT-II \\ investigators
}

\begin{abstract}
${ }^{1}$ Hospital Clínic, Hematology Department, Institut d'Investigació Biomédica August Pi i Sunyer, University of Barcelona, Barcelona, Spain; ${ }^{2}$ Department of Experimental and Clinical Medicine, University of Florence, Florence, Italy; ${ }^{3}$ The Assistance Publique - Hôpitaux de Paris, Hôpital Saint-Louis, Centre d'Investigations Cliniques and Department of Clinical Pharmacology, Université Paris Diderot, Paris, France; ${ }^{4}$ Division of Hematology and Medical Oncology, University of Leipzig, Leipzig, Germany; ${ }^{5}$ Novartis Clinical Development, Novartis Pharmaceuticals Corporation, East Hanover, NJ; ${ }^{6}$ Oncology Global Development, Novartis Pharma AG, Basel, Switzerland; ${ }^{7}$ Clinical Development, Incyte Corporation, Wilmington, DE; ${ }^{8}$ Department of Hematology, Ospedale di Circolo e Fondazione Macchi, Varese, Italy; ${ }^{9}$ Department of Hematology, Azienda Ospedaliera Ospedali Riuniti di Bergamo, Bergamo, Italy; ${ }^{10}$ Center for the Study of Myelofibrosis, Istituto Di Ricovero e Cura a Carattere Scientifico Policlinico San Matteo Foundation, Pavia, Italy; ${ }^{11}$ Department of Haematology, Guy's and St. Thomas' National Health Service Foundation Trust, London, United Kingdom; ${ }^{12}$ Hematology Unit, Cliniques Universitaires Saint-Luc and de Duve Institute, Université Catholique de Louvain, Brussels, Belgium; and ${ }^{13}$ Department of Internal Medicine, Medical University of Vienna, Vienna, Austria
\end{abstract}

- Long-term analysis of the COMFORT-II Trial shows that ruxolitinib treatment results in durable reductions in splenomegaly and is well tolerated.

- Patients randomized to ruxolitinib showed longer overall survival than those receiving the BAT.

\section{Key Points}

Ruxolitinib is a potent Janus kinase (JAK)1/JAK2 inhibitor that has demonstrated rapid reductions in splenomegaly and marked improvement in disease-related symptoms and quality of life in patients with myelofibrosis (MF). The present analysis reports the 3-year follow-up (median, 151 weeks) of the efficacy and safety of Controlled Myelofibrosis Study With Oral Janus-associated Kinase (JAK) Inhibitor Treatment-II (the COMFORT-II Trial), comparing ruxolitinib with the best available therapy (BAT) in 219 patients with intermediate-2 and high-risk MF. In the ruxolitinib arm, with continued therapy, spleen volume reductions of $\geq 35 \%$ by magnetic resonance imaging (equivalent to approximately $50 \%$ reduction by palpation) were sustained for at least 144 weeks, with the probability of $50 \%(95 \%$ confidence interval $[\mathrm{Cl}], 36-63)$ among patients achieving such degree of response. At the time of this analysis, $45 \%$ of the patients randomized to ruxolitinib remained on treatment. Ruxolitinib continues to be well tolerated. Anemia and thrombocytopenia were the main toxicities, but they were generally manageable, improved over time, and rarely led to treatment discontinuation ( $1 \%$ and $3.6 \%$ of patients, respectively). No single nonhematologic adverse event led to definitive ruxolitinib discontinuation in more than 1 patient. Additionally, patients randomized to ruxolitinib showed longer overall survival than those randomized to BAT (hazard ratio, 0.48; 95\% Cl, 0.28-0.85; log-rank test, $P=.009$ ). This trial was registered at clinicaltrials.gov as \#NCT00934544. (Blood. 2013;122(25):4047-4053)

\section{Introduction}

Myelofibrosis (MF) is a myeloproliferative neoplasm that can appear de novo (primary MF) or follow polycythemia vera (PV) or essential thrombocythemia. ${ }^{1} \mathrm{MF}$ is characterized by bone marrow fibrosis, extramedullary hematopoiesis with progressive splenomegaly, cytopenias or cytosis, and a leukoerythroblastic blood picture., ${ }^{2,3}$ Main symptoms include those derived from anemia, splenomegaly (eg, abdominal pain, early satiety), and debilitating constitutional symptoms (ie, night sweats, weight loss, and fever) leading to cachexia. ${ }^{3,4}$ Approximately $60 \%$ of patients with MF harbor the V617F mutation of the Janus kinase (JAK) 2 (JAK2) gene. However,

Submitted February 20, 2013; accepted October 20, 2013. Prepublished online as Blood First Edition paper, October 30, 2013; DOI 10.1182/blood2013-02-485888.

F.C. and A.M.V. contributed equally to this study.

The online version of this article contains a data supplement.

dysregulation of the JAK/signal transducer and activator of transcription pathway is present in all patients irrespective of their $J A K 2$ mutational status. ${ }^{5}$ Ruxolitinib is a potent JAK1/JAK2 inhibitor $^{6}$ that has demonstrated rapid reductions in splenomegaly and improved MF-related symptoms and quality of life in 2 phase 3 studies comparing ruxolitinib with placebo in the Controlled Myelofibrosis Study With Oral JAK Inhibitor Treatment (the COMFORT-I Trial) ${ }^{7}$ or best available therapy (BAT) in the Controlled Myelofibrosis Study With Oral JAK Inhibitor Treatment-II (the COMFORT-II Trial). ${ }^{8}$ Based on the results of

The publication costs of this article were defrayed in part by page charge payment. Therefore, and solely to indicate this fact, this article is hereby marked "advertisement" in accordance with 18 USC section 1734. 
these studies, ruxolitinib was approved by the US Food and Drug Administration for the treatment of intermediate- or high-risk MF and by the European Commission and Health Canada for the treatment of disease-related splenomegaly or symptoms in adult patients with MF. In addition to profound symptomatic improvement, the 2-year follow-up analysis of the COMFORT-I Trial showed a survival advantage for patients receiving ruxolitinib as compared with placebo. ${ }^{7}$

The present analysis updates the efficacy and safety findings of the COMFORT-II Trial with 3 years of follow-up and shows a relative reduction in the risk of death with ruxolitinib treatment compared with the BAT.

\section{Methods}

\section{Study design}

The COMFORT-II Trial is an open-label phase 3 study that includes patients with a diagnosis of primary MF (PMF), post-polycythemia vera MF (PPV-MF), or postessential thrombocythemia MF (PET-MF) by World Health Organization and International Working Group for Myelofibrosis Research and Treatment criteria ${ }^{9,10}$ classified as intermediate- 2 or high risk using the International Prognostic Scoring System (IPSS). ${ }^{3}$ Patients $(\mathrm{n}=219)$ were randomized $2: 1$ to receive ruxolitinib (15 or $20 \mathrm{mg}$ twice daily, based on baseline platelet count $\left[100-200\right.$ or $>200 \times 10^{9} / \mathrm{L}$, respectively]) or the BAT. The latter was at the discretion of the treating investigator, who could include any commercially available agents alone or in combination, or no active therapy, and this could be changed at any time during the treatment phase. Patients who progressed (defined as the need for splenectomy or progressive splenomegaly [ $>25 \%$ increase in spleen volume above the on-study nadir, including baseline]) were able to receive ruxolitinib in an extension phase. After the primary analysis in January 2011, the study protocol was amended (amendment 5) to allow all patients to enter the extension phase, including those who did not meet the criteria for progression. Further details of the study design and inclusion and exclusion criteria were previously described. ${ }^{8}$ The data cutoff for this analysis was December 1, 2012.

The study was designed by Incyte Corporation, approved by the institutional review boards of the respective institutions, and conducted in accordance with the principles of the Declaration of Helsinki. All patients had provided written informed consent. Data were analyzed and interpreted by the sponsor's clinical and statistical teams in collaboration with the investigators. An independent data and safety monitoring board reviewed the trial data and made recommendations regarding the continuation of the study.

\section{Efficacy analyses}

The primary and key secondary endpoints of the study were the proportion of patients achieving a $\geq 35 \%$ reduction in spleen volume from baseline at week 48 and at week 24 , respectively; assessments of spleen volume by magnetic resonance imaging were collected every 12 or 24 weeks after week 48 , depending on spleen response status. Additional secondary end points included overall survival and the duration of spleen response. A loss of spleen response was defined as an increase in spleen volume that was no longer a $\geq 35 \%$ reduction from baseline and is $>25 \%$ above the on-study nadir. Assessments of symptoms and quality of life were not collected beyond week 48 or for patients who entered the extension phase.

Efficacy results are based on an intention-to-treat analysis. For the assessment of spleen response, results are shown for the randomized and extension phases for both the ruxolitinib and the BAT arms and include patients who crossed over from BAT to ruxolitinib. For assessment of the duration of spleen response, data from the randomized and extension phases are shown for ruxolitinib-treated patients and until crossover for BATtreated patients.
Measurement of the dynamics of $J A K 2 \mathrm{~V} 617 \mathrm{~F}$ allele burden in patients with the mutation was an exploratory end point. $J A K 2 \mathrm{~V} 617 \mathrm{~F}$ allele burden was measured from blood samples using allele-specific quantitative realtime polymerase chain reaction, as described in 2006 by Levine et al, ${ }^{11}$ using an Applied Biosystems ABI 7900 real-time polymerase chain reaction (PCR) analyzer.

Overall survival was estimated using the Kaplan-Meier method, and the hazard ratio was determined using the Cox proportional hazards model stratified by baseline IPSS category. The $P$ value was determined by using the stratified log-rank test and was presented at face value without adjustment for multiple comparisons.

\section{Safety}

Safety results are summarized for the entire duration of treatment of patients randomized to ruxolitinib (ie, randomized and extension phases) for the duration of BAT until crossover for patients randomized to BAT (ie, randomized phase only), and separately after crossover for BAT patients who received ruxolitinib treatment in the extension phase. Nonhematologic adverse events (AEs) are summarized by adjusted rate per 100 patient-year exposure. AEs of special interest were defined by previously known or potential toxicities and are presented as the incidence rate by 24 -week intervals.

\section{Results}

\section{Patients}

The patient details and primary results of the study were previously reported, with shorter treatment duration (median, 50.1 weeks). ${ }^{8}$ In brief, baseline characteristics were well-balanced between treatment groups; $53 \%$ of patients had PMF, 31\% PPV-MF, and $16 \%$ PET-MF. Approximately $40 \%$ and $60 \%$ of patients in both arms were clinically confirmed as intermediate- 2 or high risk, respectively, according to the IPSS. ${ }^{3}$

Overall, $73 \%$ of patients (106 of 146) in the ruxolitinib arm and $62 \%$ ( 45 of 73 ) in the BAT arm entered the extension phase to receive ruxolitinib, and at the time of this analysis, 45\% (66 of 146) of those originally randomized to ruxolitinib remained on treatment (Table 1). The median follow-up was 151 weeks (ruxolitinib, 151 weeks; BAT, 122 weeks), and the median duration of exposure was 136 weeks to ruxolitinib (randomized and extension phases) and 45 weeks to BAT (randomized treatment only). For patients starting on $20 \mathrm{mg}$ twice daily, the median ruxolitinib daily dose remained stable to week 48 (39.7 $\mathrm{mg} /$ day) and then decreased slightly over time to week 144 ( $34.3 \mathrm{mg} /$ day). In the $15 \mathrm{mg}$ twice daily group, the median dose intensity decreased over the first 24 weeks of therapy and stabilized at approximately $20 \mathrm{mg} /$ day $(20.8 \mathrm{mg} /$ day at week 144; supplemental Figure 1; available on the Blood Web site). In the BAT arm, 24 patients (33\%) received no active treatment while on the study; of the $67 \%$ of patients who received the BAT, the most commonly used therapies were antineoplastic agents (51\%), primarily hydroxyurea $(\mathrm{n}=34)$, and glucocorticoids $(16 \%)$. Of the patients who crossed over from the BAT arm to receive ruxolitinib, $42 \%$ (19 of 45) did not receive MF-specific medication, and 58\% (26 of 45 ) were on active treatment (with 18 who received hydroxyurea).

\section{Efficacy}

Spleen response. In the ruxolitinib arm, 97\% of patients (132 of 136) with post-baseline assessments experienced a clinical benefit with some degree of reduction in spleen volume at any time of the study, and 51\% (75 of 146) achieved a protocol-defined spleen 
Table 1. Patient disposition at 3-year follow-up of the COMFORT-II Trial

\begin{tabular}{|c|c|c|c|}
\hline n (\%) & $\begin{array}{c}\text { Ruxolitinib } \\
(n=146)\end{array}$ & $\begin{array}{c}\text { BAT } \\
(n=73)\end{array}$ & $\begin{array}{c}\text { Ruxolitinib } \\
\text { after } \\
\text { BAT }(n=45)\end{array}$ \\
\hline Still on treatment & $66(45.2)$ & 0 & - \\
\hline Discontinued & $80(54.8)$ & $28(38.4)$ & - \\
\hline Crossed over* & - & $45(61.6)$ & - \\
\hline $\begin{array}{l}\text { After qualifying progression } \\
\text { event }\end{array}$ & - & $26(35.6)$ & - \\
\hline After protocol amendment 5 & - & $13(17.8)$ & - \\
\hline Othert & - & $6(8.2)$ & - \\
\hline Still on treatment after crossover & - & - & $22(48.9)$ \\
\hline Discontinued after crossover & - & - & $23(51.1)$ \\
\hline \multicolumn{4}{|l|}{$\begin{array}{l}\text { Primary reasons for } \\
\text { discontinuation }\end{array}$} \\
\hline $\mathrm{AE}$ & $24(16.4)$ & $5(6.8)$ & $6(13.3)$ \\
\hline Consent withdrawn & $9(6.2)$ & $9(12.3)$ & 0 \\
\hline Protocol deviation & $2(1.4)$ & 0 & $5(11.1)$ \\
\hline Disease progression & $22(15.1)$ & $4(5.5)$ & $6(13.3)$ \\
\hline $\begin{array}{l}\text { Noncompliance with study } \\
\text { medication }\end{array}$ & $3(2.1)$ & 0 & $1(2.2)$ \\
\hline $\begin{array}{l}\text { Noncompliance with study } \\
\text { procedures }\end{array}$ & 0 & $1(1.4)$ & 0 \\
\hline $\begin{array}{l}\text { Unsatisfactory therapeutic } \\
\text { effect }\end{array}$ & $5(3.4)$ & 0 & $1(2.2)$ \\
\hline Other $\ddagger$ & $15(10.3)$ & $9(12.3)$ & $4(8.9)$ \\
\hline
\end{tabular}

*Patients randomized to the BAT arm could crossover to ruxolitinib treatment at any time during the study upon a protocol-defined progression event. Patients in the ruxolitinib arm who had a protocol-defined progression event could continue receiving ruxolitinib in the extension phase at any time during the study if, in the investigator's opinion, they were still receiving a benefit from ruxolitinib treatment. Progression events that qualified for the crossover and extension phases included the need for splenectomy and progressive splenomegaly as defined by a $25 \%$ increase in spleen volume compared with the on-study nadir (including baseline). After the primary analysis, the study protocol was amended (amendment 5) in January 2011 to allow all patients to enter the extension phase, including those who did not meet the criteria for progression.

†Six patients crossed over from BAT to ruxolitinib prior to protocol amendment 5 without experiencing qualifying progression events (5 patients discontinued due to the protocol deviation and 1 patient discontinued due to other reason).

$\neq$ Other reasons for discontinuation in the ruxolitinib arm included patients who underwent stem cell transplant $(n=5)$, interruption of study medication for $>8$ weeks $(n=2)$, lack of efficacy $(n=2)$, meeting protocol-defined imaging discontinuation criteria $(n=2)$, investigator decision $(n=1)$, diagnosis of lung cancer with the start of chemotherapy treatment $(n=1)$, unspecified safety event $(n=1)$, and modest spleen response $(n=1)$. Other reasons in the BAT arm included stem cell transplant $(n=2)$, investigator decision $(n=2)$, patient decision $(n=2)$, splenic irradiation $(n=1)$, hematemesis and thrombocytopenia $(n=1)$, unwillingness to undergo magnetic resonance imaging $(n=1)$, enrollment in a ruxolitinib compassionate use program $(n=1)$, splenectomy $(n=1)$, initiating treatment with hydroxyurea $(n=1)$, and thrombocytopenia as sign of disease progression $(n=1)$.

response of a $\geq 35 \%$ spleen reduction. Six patients achieved the spleen response after the primary analysis at week 48 and are included in this analysis. BAT patients who crossed over to ruxolitinib had reductions in spleen volume after crossover (Figure 1). One patient in the BAT arm achieved a $\geq 35 \%$ reduction in spleen volume at week 15 but did not maintain the response at week 48 . This patient crossed over to the ruxolitinib arm without having a protocol-defined progression event and was excluded from the per-protocol analysis. The spleen reductions of $\geq 35 \%$ obtained with ruxolitinib were sustained with continued therapy (median duration not yet reached; Figure 2). The Kaplan-Meier estimated probabilities of maintaining the spleen response at weeks 48 and 144 were $73 \%$ (95\% CI, 61-82) and 50\% (95\% CI, 36-63), respectively.

JAK2 V617F allele burden. At baseline, 110 patients (76\%) in the ruxolitinib arm and 49 patients $(71 \%)$ in the BAT arm were determined to be $J A K 2$ V617F-positive, and the median allele burdens were $84.5 \%$ (range, $5 \%$ to $96 \%$ ) and $81 \%$ (range, $1 \%$ to
$95 \%$ ) in the ruxolitinib and BAT arms, respectively. Overall, patients in the ruxolitinib arm had a median change from baseline of $-7.0 \%$ at week 48 (range, $-47 \%$ to $8 \% ; n=69$ ) and $-8.0 \%$ at week 72 (range, $-51 \%$ to $15 \% ; \mathrm{n}=53$ ), whereas those in the BAT arm had no median change from baseline at either time point (week 48: range, $-13 \%$ to $10 \% ; \mathrm{n}=22$ ) (week 72 : range, $-4 \%$ to $12 \%$; $\mathrm{n}=8$ ). More patients in the ruxolitinib arm had allele burden reductions of $\geq 10 \%$ compared with BAT patients at week 48 (42\% [29 of 69] vs $9 \%$ [2 of 22]) and at week 72 (40\% [21 of 53] vs $0 \%)$. Of these, 15 of 69 ruxolitinib-treated patients (22\%) had reductions of $\geq 20 \%$ at week 48 (median, $-32 \%$; range, $-47 \%$ to $-21 \%$ ), and $83 \%$ of patients maintained their reduction at the last time point of week 72 (median, $-40 \%$; range, $-51 \%$ to $-20 \%$ ). Allele burden reductions in most patients treated with ruxolitinib were gradual over the course of the study, and were similar across MF subtypes (supplemental Figure 2). Among patients who achieved $\geq 20 \%$ of a reduction in allele burden, $39 \%$ had PMF, $39 \%$ had PPV-MF, and $22 \%$ had PET-MF, similar to the overall study population. No patient receiving the BAT had allele burden reductions of $\geq 20 \%$ at either time point. Additionally, a higher proportion of patients with $\geq 20 \%$ of an allele burden reduction achieved a protocol-defined spleen response with ruxolitinib compared with those with $<10 \%$ of a reduction at both week 48 (73\% vs $19 \%$; odds ratio, 4.6) and week 72 (69\% vs $22 \%$; odds ratio, 4.2) (supplemental Figure 3).

\section{Safety}

Nonhematologic toxicity was primarily grade $1 / 2$, and the overall pattern and frequency of the most common AEs (ie, observed in $\geq 10 \%$ of patients) did not change with longer treatment. When adjusted for exposure to study medication (Table 2), the rates of nonhematologic AEs generally decreased with longer-term ruxolitinib treatment and were lower than in the BAT. Additionally, no single nonhematologic AE led to treatment discontinuation in more than 1 patient.

The AEs of special interest included anemia, thrombocytopenia, leukopenia, bleeding, infections, thromboembolic events, elevated transaminase levels, increased systolic blood pressure, weight gain, and secondary malignancies. All grade AEs of special interest that occurred in $\geq 5 \%$ of patients in any interval and in any preferred term are shown by 6 -month intervals in Table 3 . The rate of these events generally decreased with longer exposure to ruxolitinib treatment, with the highest rates occurring within the first 6 months of treatment. Over the entire course of the study, 2 patients $(1.4 \%)$ in the ruxolitinib arm had tuberculosis.

New or worsening AEs that occurred within 28 days after ruxolitinib discontinuation were also recorded (supplemental Table 1). The rate and severity of these AEs did not differ from what was reported for AEs occurring for patients on treatment, or they were consistent with those expected for patients receiving no treatment. Overall, $30.1 \%$ of patients (31 of 103) reported experiencing AEs after discontinuation of ruxolitinib treatment (including after crossover from BAT), and the most common were infections (13.6\% [14 of 103]) and thrombocytopenia (9.7\% [10 of 103]). The majority of safety events occurred in only 1 patient each; events occurring after discontinuation in $>1$ patient in either the ruxolitinib arm or after crossover from the BAT included preferred terms of thrombocytopenia $(n=10)$, pneumonia $(n=3)$, anemia $(n=2)$, and hypoalbuminemia $(\mathrm{n}=2)$.

The most common laboratory abnormalities were anemia and thrombocytopenia. However, the majority of these AEs were grade 


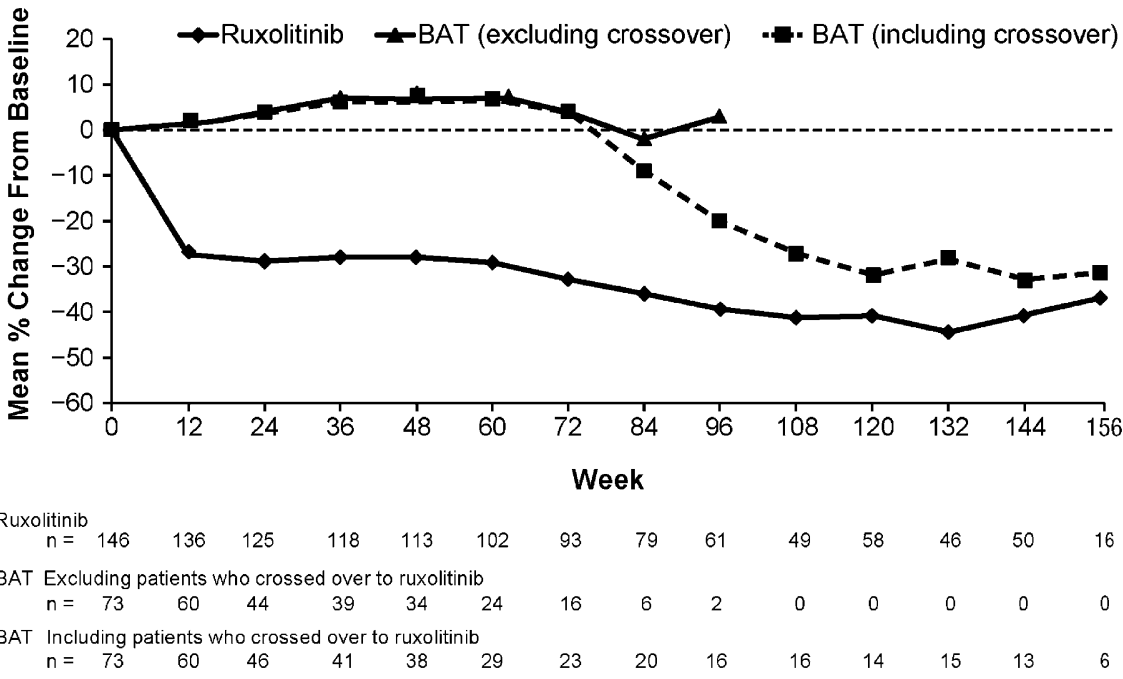

Figure 1. Mean percentage change in spleen volume over time. Assessments of changes in spleen volume in the BAT arm were determined based on the patients' original baseline spleen volume and not from the time of crossover. Patients crossed over from the BAT arm to receive ruxolitinib over the course of approximately 6 to 8 months.

1/2 (supplemental Table 2) and rarely led to treatment discontinuation among patients who received ruxolitinib, including extension phase and crossover from BAT. Thus, only 2 patients (1\%) discontinued the treatment due to anemia or hemoglobin decreases and 7 (3.6\%) due to thrombocytopenia or platelet count decreases. In the ruxolitinib arm, mean hemoglobin levels decreased over the first 12 weeks of treatment and then recovered to levels similar to those in the BAT patients and remained $>10 \mathrm{~g} / \mathrm{dL}$ from week 24 onward (Figure 3). Similarly, the transfusion rate was slightly higher in the ruxolitinib arm over the first 24 weeks, but declined thereafter to a rate similar to that in the BAT arm (supplemental Figure 4).

During treatment or within 28 days after discontinuation of study medication, there were 13 deaths $(8.9 \%)$ in the ruxolitinib arm, 4 deaths $(5.5 \%)$ in the BAT arm during randomized treatment, and $1(2.2 \%)$ after crossover from BAT at the time of data cutoff. A patient can have multiple causes of death and be included in $\geq 1$ category; in the ruxolitinib arm, each cause of death category included only 1 patient. Causes of death in the ruxolitinib arm during randomized treatment included cardiac arrest, cardiac failure, intestinal perforation, retroperitoneal hemorrhage, disease progression, multiorgan failure, hepatic failure, portal vein thrombosis, endocarditis, septic shock, postoperative respiratory distress, and cerebral hemorrhage (1 patient each). Causes of death during ruxolitinib treatment in the extension phase included enterococcal sepsis, febrile infection, pneumonia, cerebrovascular accident, and depressed level of consciousness (1 patient each). Causes of death in the BAT arm during randomized treatment included respiratory failure $(n=2)$, cardiac failure $(n=1)$, and renal impairment $(\mathrm{n}=1)$; causes of death after crossover from BAT to ruxolitinib included ascites and Klebsiella sepsis (both in the same patient). At the time of the primary analysis at 48 weeks, 2 cases of transformation to acute leukemia (defined as a peripheral blood blast count of $\geq 20 \%$ sustained for $\geq 8$ weeks or a bone marrow blast count of $\geq 20 \%$ ) were reported in BAT-treated patients. ${ }^{8}$ With this longer follow-up, 5 patients $(3.4 \%)$ in the ruxolitinib arm and 4 patients $(5.5 \%)$ in the BAT arm experienced leukemic transformation ( 1 of which was recorded in the safety database as a serious $\mathrm{AE})$.

\section{Overall survival analysis}

Since the last report (median, 112 weeks), ${ }^{12}$ an additional 9 and 6 deaths were reported overall in the ruxolitinib and BAT arms, resulting in a mortality rate of $19.9 \%$ (29 of 146) and 30.1\% (22 of 73) of patients, respectively; therefore, the median survival time has not yet been reached in either arm (Figure 4). Among the patients who died in the BAT arm (22 in total), only 4 patients did not receive any MF-specific medication, and 18 had active treatment (patients may have received $\geq 1$ BAT medications at any given time). Patients randomized to ruxolitinib showed longer overall survival than those randomized to BAT; there was a $52 \%$ reduction in the risk of death in the ruxolitinib arm compared with the BAT arm
Figure 2. Duration of spleen response. Includes randomized and extension phases for patients randomized to ruxolitinib; crossover patients are not included. Duration of maintenance was estimated only for patients who achieved $a \geq 35 \%$ reduction in spleen volume. The start date was defined as the first spleen volume measurement that was a $\geq 35 \%$ reduction from baseline (minimum of 12 weeks [ie, first on-treatment assessment by magnetic resonance imaging]), and the end date was the first measurement that was no longer a $\geq 35 \%$ reduction and that was a $>25 \%$ increase above the nadir.

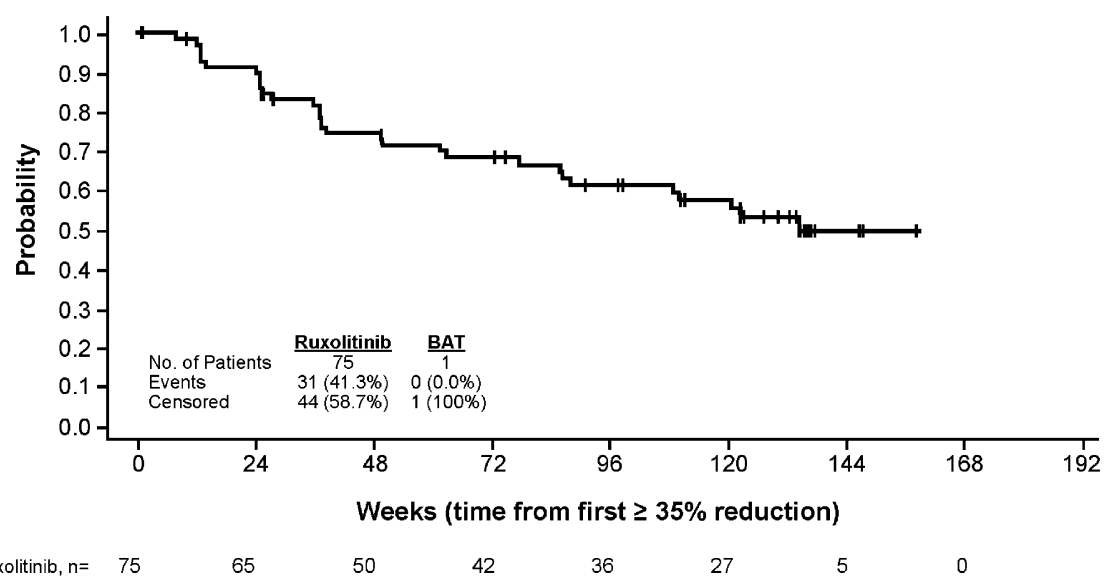


Table 2. Nonhematologic AEs regardless of study drug

\begin{tabular}{|c|c|c|c|c|}
\hline $\begin{array}{l}\text { Adjusted } \\
\text { rate per } 100 \\
\text { patient-year } \\
\text { exposure }\end{array}$ & $\begin{array}{l}\text { Ruxolitinib } \\
\text { randomized } \\
(n=146)\end{array}$ & $\begin{array}{c}\text { Ruxolitinib } \\
\text { randomized } \\
+ \\
\text { extension } \\
(n=146)\end{array}$ & $\begin{array}{c}\text { BAT } \\
\text { randomized } \\
(n=73)\end{array}$ & $\begin{array}{c}\text { Ruxolitinib } \\
\text { crossover } \\
(\mathrm{n}=45)\end{array}$ \\
\hline $\begin{array}{r}\text { Patient-year } \\
\text { exposure }\end{array}$ & 170.12 & 304.87 & 66.98 & 44.95 \\
\hline Peripheral edema & $20.0(0)$ & $17.4(0)$ & $31.4(1.5)$ & $17.8(2.2)$ \\
\hline Diarrhea & $22.3(1.2)$ & $15.4(0.7)$ & $19.4(0)$ & $20.0(0)$ \\
\hline Asthenia & $16.5(2.4)$ & $11.5(1.6)$ & $13.4(1.5)$ & $17.8(2.2)$ \\
\hline Dyspnea & $14.1(1.2)$ & $11.2(1.3)$ & $22.4(4.5)$ & $20.0(2.2)$ \\
\hline Pyrexia & $12.9(1.8)$ & $11.5(1.3)$ & $10.5(0)$ & $13.3(0)$ \\
\hline Fatigue & $13.5(0.6)$ & $11.2(0.7)$ & $11.9(0)$ & $13.3(2.2)$ \\
\hline Nasopharyngitis & $15.9(0)$ & $11.8(0)$ & $14.9(0)$ & $8.9(0)$ \\
\hline Bronchitis & $10.6(1.2)$ & $11.5(1.3)$ & $9.0(1.5)$ & $6.7(0)$ \\
\hline Cough & $12.9(0)$ & $10.5(0)$ & $17.9(1.5)$ & $11.1(2.2)$ \\
\hline Arthralgia & $11.2(1.2)$ & $8.9(0.7)$ & $10.5(0)$ & $13.3(2.2)$ \\
\hline Weight gain & $13.5(1.8)$ & $9.5(1.0)$ & $1.5(0)$ & $8.9(0)$ \\
\hline Nausea & $12.3(0.6)$ & $8.9(0.3)$ & $10.5(0)$ & $8.9(0)$ \\
\hline Pain in extremity & $10.0(0.6)$ & $7.2(0.3)$ & $6.0(0)$ & $20.0(0)$ \\
\hline Headache & $10.6(1.2)$ & $6.9(0.7)$ & $6.0(0)$ & $15.6(0)$ \\
\hline Back pain & $10.6(1.8)$ & $7.5(1.3)$ & $14.9(0)$ & $6.7(0)$ \\
\hline Insomnia & $5.3(0)$ & $3.9(0)$ & $10.5(0)$ & $8.9(0)$ \\
\hline Abdominal pain & $10.0(2.9)$ & $6.6(1.6)$ & $19.4(4.5)$ & $6.7(2.2)$ \\
\hline Epistaxis & $7.6(0)$ & $5.2(0.7)$ & $7.5(0)$ & $11.1(0)$ \\
\hline Pruritus & $5.3(0)$ & $5.2(0)$ & $19.4(0)$ & $8.9(0)$ \\
\hline
\end{tabular}

Data are presented as adjusted rates for all grades, with grades $3 / 4$ given in parentheses. Relationship adjusted for patient-year exposure (rate of $\geq 10$ per 100 patient-years in either arm) by preferred term. AEs regardless of study drug relationship occurring $>28$ days after discontinuation of study medication are not summarized. A patient with multiple occurrences of an $A E$ under 1 treatment is counted only once in the $A E$ category for that treatment.

(hazards ratio, $0.48 ; 95 \% \mathrm{CI}, 0.28-0.85 ; P=.009$ ). The KaplanMeier estimated probability of survival at 144 weeks was $81 \%$ in the ruxolitinib arm and $61 \%$ in the BAT arm.

\section{Discussion}

This longer follow-up of the COMFORT-II Trial shows that the reductions in the spleen volume observed at the time of the initial analysis in the patients assigned to the ruxolitinib arm were maintained with continued treatment in a substantial proportion of the patients. Thus, the median duration of a spleen response has not yet been reached after 3 years, although only a few more events are required. In this sense, it must be noted that the patients in this analysis were in the advanced stages of MF (intermediate- 2 and high-risk groups), as required for inclusion in this study.

As expected, and given its mechanism of action as a JAK1 and JAK2 inhibitor, the most common AEs associated with ruxolitinib were anemia and thrombocytopenia. However, most events were grade $1 / 2$ and rarely led to definitive treatment discontinuation. Thus, among patients receiving ruxolitinib, including the extension phase and crossover from BAT, only 2 patients discontinued due to anemia and 7 due to thrombocytopenia. In addition, mean hemoglobin levels in the ruxolitinib arm decreased at the beginning of treatment and recovered to levels similar to those of the BAT patients from week 24 onward, whereas the transfusion rate was slightly higher in the ruxolitinib arm over the first 24 weeks and declined thereafter to a rate similar to that in the BAT arm. Concerning nonhematologic toxicity, an increased frequency of infections was observed under ruxolitinib. However, these complications were rarely severe and tended to decrease over time. Overall, no new safety signals have emerged with this longer-term ruxolitinib treatment compared with the initial analysis.

The survival advantage with ruxolitinib treatment compared with BAT in this update confirms the results obtained in a previous analysis performed after a median follow-up of 112 weeks. ${ }^{12}$ This advantage is noteworthy, taking into account the expected survival of the patient population included in the study. Indeed, the expected median survival for patients with intermediate- 2 risk MF (ie, $60 \%$ of the patients in the study) is 48 months and 27 months for patients with high-risk MF. ${ }^{3}$ Therefore, given the composition of the study population, the expected median survival would have slightly exceeded the 3 years of the current follow-up. In this sense, a difference in survival between the 2 arms may not have been observed at earlier time points for several reasons. First, as mentioned before, the MF population included in the study had an expected median survival that is less than the median duration of follow-up for most patients, and therefore, the death events were expected to occur not at the beginning of the study but later. Second, the 2:1 randomization means there are fewer potential events in the comparator arm. In addition, a considerable number of patients in the BAT arm were censored prior to 48 weeks $(24.7 \%$ of patients in the BAT arm and $9.6 \%$ of patients in the ruxolitinib arm). ${ }^{12}$ Most patients in the BAT arm

Table 3. AEs of special interest by 6-month intervals

\begin{tabular}{|c|c|c|c|c|c|c|c|}
\hline \multirow{2}{*}{$\begin{array}{l}\text { Ruxolitinib randomized + } \\
\text { extension, \% }\end{array}$} & \multicolumn{7}{|c|}{ Week } \\
\hline & $0-24(n=146)$ & $24-48(n=134)$ & $48-72(n=116)$ & $72-96(n=101)$ & $96-120(n=93)$ & $120-144(n=81)$ & $144-168(n=72)$ \\
\hline Anemia* & 34.9 & 12.7 & 8.6 & 13.9 & 8.6 & 7.4 & 8.3 \\
\hline Thrombocytopenia† & 43.2 & 22.4 & 15.5 & 12.9 & 10.8 & 12.3 & 2.8 \\
\hline Bleeding & 17.1 & 14.2 & 9.5 & 11.9 & 7.5 & 9.9 & 6.9 \\
\hline Epistaxis & 6.8 & 1.5 & 0.9 & 4.0 & 0 & 1.2 & 1.4 \\
\hline Hematoma & 5.5 & 4.5 & 3.4 & 1.0 & 0 & 2.5 & 1.4 \\
\hline Infections & 50.0 & 35.1 & 37.9 & 25.7 & 43.0 & 33.3 & 25.0 \\
\hline Bronchitis & 3.4 & 6.7 & 8.6 & 3.0 & 10.8 & 4.9 & 4.2 \\
\hline Gastroenteritis & 5.5 & 3.0 & 0.9 & 1.0 & 2.2 & 1.2 & 0 \\
\hline Nasopharyngitis & 13.7 & 5.2 & 7.8 & 4.0 & 10.8 & 3.7 & 4.2 \\
\hline Urinary tract infection & 4.8 & 2.2 & 5.2 & 4.0 & 5.4 & 3.7 & 2.8 \\
\hline Weight gain & 8.2 & 8.2 & 5.2 & 5.0 & 2.2 & 0 & 0 \\
\hline
\end{tabular}

Data are presented as the percentage of patients. All grades for $\geq 5 \%$ of patients in any preferred term within the standardized MedDRA queries (SMQ) and any interval. Patients are counted only once in each category and time interval. AEs of special interest include anemia, thrombocytopenia, leukopenia, bleeding, infections, thromboembolic events, weight gain, elevated transaminase levels, increased systolic blood pressure, and secondary malignancies.

*Includes preferred terms of anemia and normochromic normocytic anemia; the SMQ term is erythropenia.

†Includes preferred terms of thrombocytopenia and platelet count decreased. 


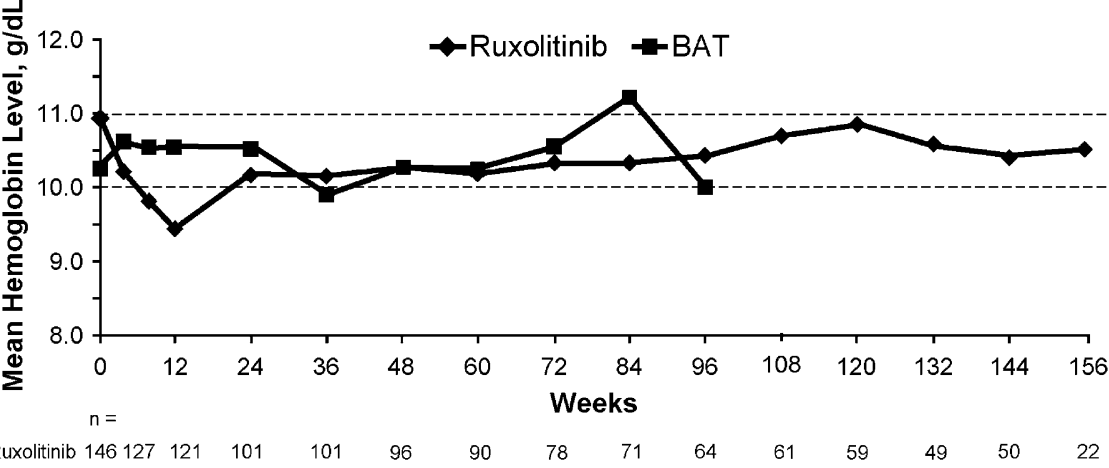

Figure 3. Hemoglobin levels and platelet counts over time. Ruxolitinib includes both the randomized and extension phases; BAT includes the randomized phase only and not the assessments after crossover.

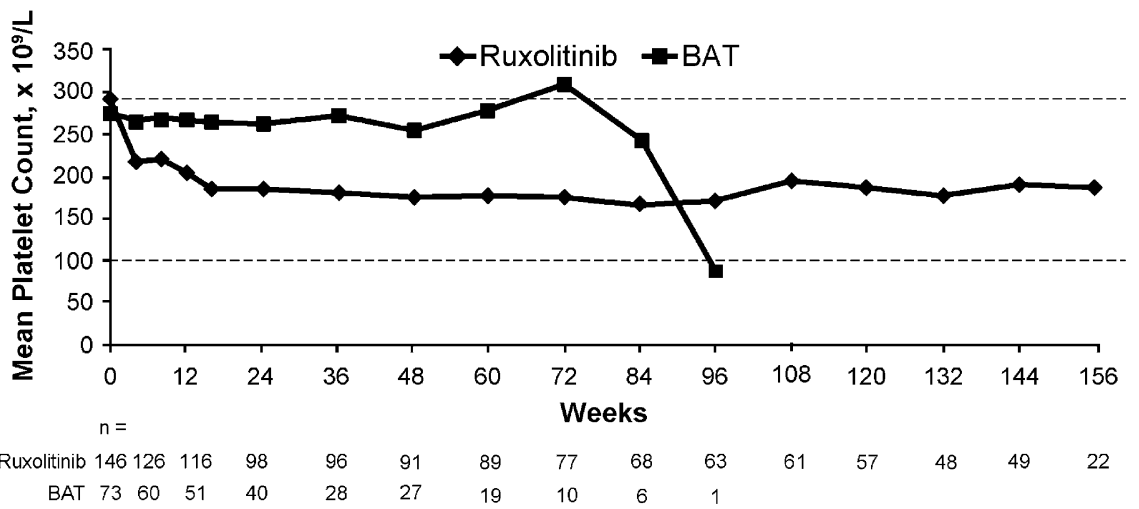

who were lost to follow-up discontinued because of consent withdrawal, likely because of a lack of benefit from study medication, whereas the lower proportion of patients lost to follow-up in the ruxolitinib arm was primarily related to intolerance and, to a lesser degree, lack of benefit from study medication. In addition, patients were allowed to crossover from the BAT arm to receive ruxolitinib and, in an intention-to-treat analysis, these patients are considered to be under BAT treatment. The late separation observed between the Kaplan-Meier curves (after 72 weeks) may reflect outcome differences incurred within the first 48 weeks of the study, including improvements in splenomegaly, constitutional symptoms, MF-related symptoms, and patients' general condition in ruxolitinib-treated patients, and worsening in patients receiving the BAT. Despite these factors, there was a relative reduction in the risk of death with ruxolitinib treatment compared with conventional therapies.

One of the ways that ruxolitinib may improve survival is by ameliorating the patients' nutritional status and general healthrelated quality of life, thereby making patients less vulnerable and increasing their ability to withstand multiple causes of mortality. This hypothesis is supported by a post hoc analysis of survival outcomes in the COMFORT-I Trial, in which patients who had reductions in cachexia and larger improvements in cholesterol
Figure 4. Kaplan-Meier analysis of overall survival in the COMFORT-II Trial. In this intention-to-treat analysis of overall survival, patients who crossed over from the BAT arm to receive ruxolitinib are included in the BAT group.

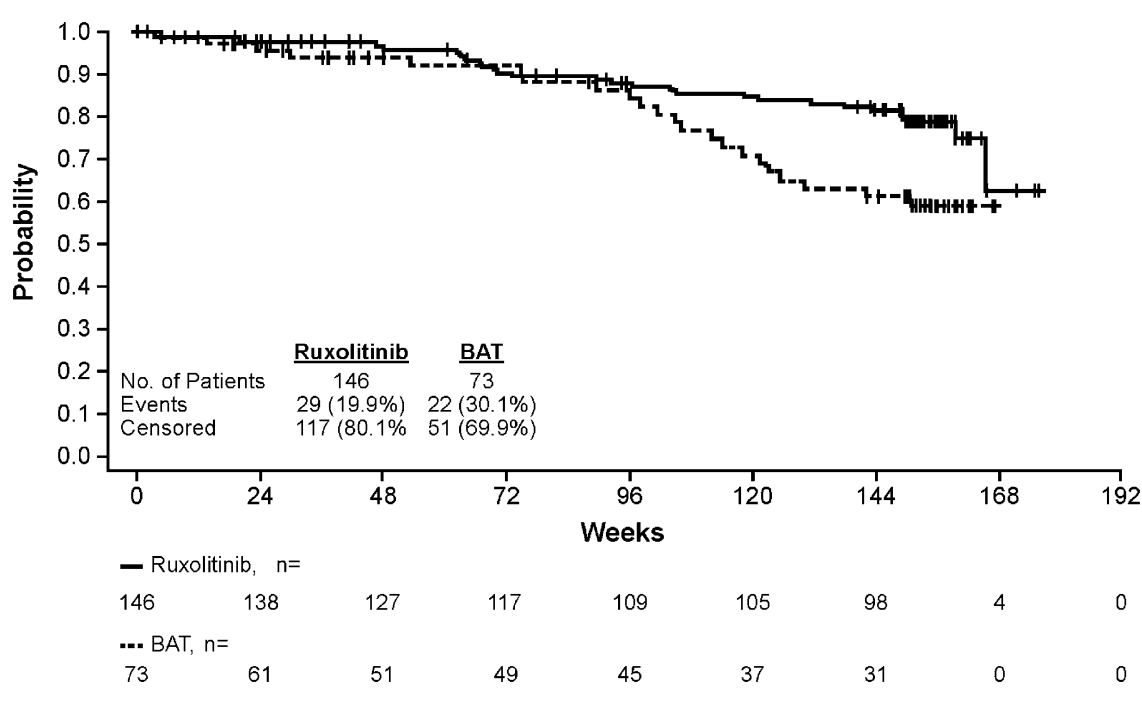

Hazard ratio, $0.48 ; 95 \% \mathrm{Cl}, 0.28-0.85$; log-rank $P=.009$; unadjusted for multiple comparisons. 
levels had significantly longer survival than those who had lesser improvements. ${ }^{13}$ Another potential mechanism would be the reduction or normalization of circulating levels of inflammatory cytokines, which are integral to MF progression and likely contribute to diseaserelated symptoms. ${ }^{14}$ Additionally, a recent report by Kvasnicka et $\mathrm{al}^{15}$ has retrospectively analyzed the fibrosis scores among patients receiving ruxolitinib in the phase $1 / 2$ study compared with those in a control group of patients treated with the BAT, showing stabilization or improvement in the fibrosis after 24 and 48 months under ruxolitinib and worsening in the fibrosis over the same time in patients receiving the BAT. This hypothesis, however, requires further exploration, including the analysis of the correlations between the bone marrow changes and the evolution of the clinichematologic findings reflecting the MF activity in the context of JAK1/JAK2 inhibitor treatment.

In conclusion, ruxolitinib provided rapid and durable reductions in splenomegaly in MF that were sustained for 3 years of treatment in the majority of patients in the COMFORT-II Trial. Ruxolitinib continues to be well tolerated, with nearly half of the patients remaining on the study. Additionally, the present results suggest a relative reduction in the risk of death with ruxolitinib compared with BAT, confirming the survival advantage observed in the previous update of the COMFORT-II Trial. ${ }^{12}$

\section{Acknowledgments}

Financial support for medical editorial assistance was provided by Novartis Pharmaceuticals. We thank John Togneri for his medical editorial assistance with this manuscript.

\section{Authorship}

Contribution: All authors drafted and approved the manuscript. Conflict-of-interest disclosure: F.C. has participated in advisory boards for Novartis, Sanofi-Aventis, Celgene, AOP Orphan Pharmaceuticals, Pfizer, and Teva, and has participated in speakers bureaus for Novartis and Bristol-Myers Squibb. J.-J.K. has participated in advisory boards for Novartis, Shire, and Incyte. H.K.A.-A. has acted as a consultant for Novartis, and has received research funding from Celgene and honoraria from Novartis and Celgene. V.S. is employed by and owns stock in Novartis. M.M. is employed by and owns stock in Novartis. D.S.H. is employed by Incyte. R.S.L. is employed by and owns stock in Incyte. F.P. has participated in advisory boards for Novartis, Celgene, and SanofiAventis, and has received honoraria from Novartis, Celgene, and Sanofi-Aventis. T.B. has received honoraria from Novartis. C.N.H. has received honoraria from Novartis, YM BioSciences, SanofiAventis, and Shire, has received research funding from Novartis and Shire, has acted as a consultant to YM BioSciences, and has participated in speakers bureaus for Novartis. A.M.V. has participated in advisory boards for Novartis. L.K. has acted as a consultant and participated in advisory boards for Novartis. H.G. has acted as a consultant for Novartis, Celgene, and AOP Orphan Pharmaceuticals AG, has received research funding from Novartis and Celgene, and is a member of speakers bureaus for Novartis, Celgene, and AOP Orphan Pharmaceuticals AG. The remaining authors declare no competing financial interests.

Correspondence: Francisco Cervantes, Hospital Clínic, Hematology Department, IDIBAPS, University of Barcelona, Villarroel 170, Barcelona, 08036 Spain; e-mail: fcervan@clinic.ub.es.

\section{References}

1. Tefferi A. Essential thrombocythemia, polycythemia vera, and myelofibrosis: current management and the prospect of targeted therapy. Am J Hematol. 2008;83(6):491-497.

2. Vannucchi AM. Management of myelofibrosis. Hematology Am Soc Hematol Educ Program. 2011;2011:222-230.

3. Cervantes F, Dupriez B, Pereira A, et al. New prognostic scoring system for primary myelofibrosis based on a study of the International Working Group for Myelofibrosis Research and Treatment. Blood. 2009;113(13):2895-2901.

4. Mesa RA, Schwager S, Radia D, et al. The Myelofibrosis Symptom Assessment Form (MFSAF): an evidence-based brief inventory to measure quality of life and symptomatic response to treatment in myelofibrosis. Leuk Res. 2009; 33(9):1199-1203.

5. Vainchenker W, Delhommeau F, Constantinescu $\mathrm{SN}$, Bernard OA. New mutations and pathogenesis of myeloproliferative neoplasms. Blood. 2011;118(7):1723-1735

6. Quintás-Cardama A, Vaddi K, Liu P, et al. Preclinical characterization of the selective JAK1/2 inhibitor INCB018424: therapeutic implications for the treatment of myeloproliferative neoplasms. Blood. 2010;115(15):3109-3117.
7. Verstovsek S, Mesa RA, Gotlib J, et al. A doubleblind, placebo-controlled trial of ruxolitinib for myelofibrosis. N Engl J Med. 2012;366(9): 799-807.

8. Harrison C, Kiladjian JJ, Al-Ali HK, et al. JAK inhibition with ruxolitinib versus best available therapy for myelofibrosis. N Engl J Med. 2012; 366(9):787-798.

9. Vardiman JW, Thiele J, Arber DA, et al. The 2008 revision of the World Health Organization (WHO) classification of myeloid neoplasms and acute leukemia: rationale and important changes. Blood. 2009;114(5):937-951.

10. Barosi G, Mesa RA, Thiele J, et al; International Working Group for Myelofibrosis Research and Treatment (IWG-MRT). Proposed criteria for the diagnosis of post-polycythemia vera and post-essential thrombocythemia myelofibrosis: a consensus statement from the International Working Group for Myelofibrosis Research and Treatment. Leukemia. 2008;22(2):437-438.

11. Levine RL, Belisle $\mathrm{C}$, Wadleigh $\mathrm{M}$, et al. $\mathrm{X}$-inactivation-based clonality analysis and quantitative JAK2V617F assessment reveal a strong association between clonality and JAK2V617F in PV but not ET/MMM, and identifies a subset of JAK2V617F-negative ET and MMM patients with clonal hematopoiesis. Blood. 2006; 107(10):4139-4141.

12. Cervantes F, Kiladjian J, Niederwieser D, et al. Long-term efficacy, safety, and survival findings from COMFORT-II, a phase 3 study comparing ruxolitinib with best available therapy for the treatment of myelofibrosis. [abstract] Blood. 2012; 120(21). Abstract 801.

13. Mesa RA, Verstovsek S, Gupta V, et al. Improvement in weight and total cholesterol and their association with survival in ruxolitinib-treated patients with myelofibrosis from COMFORT-I. [abstract] Blood. 2012;120(21). Abstract 1733.

14. Hasselbalch HC. Perspectives on chronic inflammation in essential thrombocythemia, polycythemia vera, and myelofibrosis: is chronic inflammation a trigger and driver of clonal evolution and development of accelerated atherosclerosis and second cancer? Blood. 2012; 119(14):3219-3225.

15. Kvasnicka HM, Thiele J, Bueso-Ramos C, et al. Long-term intervention effects on bone marrow morphology in myelofibrosis: patients treated with ruxolitinib and best available therapy [abstract]. Haematologica. 2013;98(suppl 1):249. Abstract S591. 


\section{( blood}

2013 122: 4047-4053

doi:10.1182/blood-2013-02-485888 originally published online October 30, 2013

\section{Three-year efficacy, safety, and survival findings from COMFORT-II, a phase 3 study comparing ruxolitinib with best available therapy for myelofibrosis}

Francisco Cervantes, Alessandro M. Vannucchi, Jean-Jacques Kiladjian, Haifa Kathrin Al-Ali, Andres Sirulnik, Viktoriya Stalbovskaya, Mari McQuitty, Deborah S. Hunter, Richard S. Levy, Francesco Passamonti, Tiziano Barbui, Giovanni Barosi, Claire N. Harrison, Laurent Knoops and Heinz Gisslinger

Updated information and services can be found at:

http://www.bloodjournal.org/content/122/25/4047.full.html

Articles on similar topics can be found in the following Blood collections

Clinical Trials and Observations (4244 articles)

Free Research Articles (3564 articles)

Myeloid Neoplasia (1446 articles)

Information about reproducing this article in parts or in its entirety may be found online at:

http://www.bloodjournal.org/site/misc/rights.xhtml\#repub_requests

Information about ordering reprints may be found online at:

http://www.bloodjournal.org/site/misc/rights.xhtml\#reprints

Information about subscriptions and ASH membership may be found online at:

http://www.bloodjournal.org/site/subscriptions/index.xhtml

Blood (print ISSN 0006-4971, online ISSN 1528-0020), is published weekly by the American Society of Hematology, 2021 L St, NW, Suite 900, Washington DC 20036.

Copyright 2011 by The American Society of Hematology; all rights reserved. 